\section{Mesoscopic Investigation of the Heterogeneities Induced by Channel-Die Compression}

Experimental techniques were applied to study the heterogeneities of deformation of metals at the mesoscopic scale (typically $100 \mu \mathrm{m}$ in the present case). The first are fiducial carbon grids that are transferred on to the surface of the test-pieces. Here, they were used on single and polycrystals deformed in channel-die compression. They prove efficient for strains above 1. They bring out the role of the corners of the samples, which trigger bands of deformation that grow in importance as the compression goes on. They put in evidence the mesoheterogeneities that appear in the mechanical behavior of a few highly symmetric orientations such as cube. The second technique is the use of microfocused X-rays, which give the crystallographic orientation at the same scale of $100 \mu \mathrm{m}$ and can work in the presence of the carbon grids even when there is considerable strain hardening. The gradients found in the lattice rotations are far less pronounced than the sharp localizations in the displacement field. This highlights the importance of the rotations due to the activity of the slip systems.

Keywords: fiducial grids, microfocus X-rays, mesoscopic scale, plasticity
Michel G. Darrieulat

Ecole Nationale Supérieure des Mines de Saint-Etienne,

Centre "Sciences des Matériaux et des Structures,"

UMR CNRS No. 5146 ,

158 cours Fauriel,

42023 Saint-Etienne, Cedex 2, France e-mail: darrieulat@emse.fr

Khalid Zhani

Faculté des Sciences et Techniques de Tanger, Université Abdelmalek Essâadi,

B.P. 416 ,

Tanger, Morocco

e-mail: k_zhani@hotmail.com

René-Yves Fillit Ecole Nationale Supérieure des Mines de Saint-Etienne,

Centre "Sciences des Matériaux et des Structures,"

UMR CNRS No. 5146,

158 cours Fauriel,

42023 Saint-Etienne, Cedex 2, France e-mail: fillit@emse.fr

Ahmed Chenaoui

Faculté des Sciences et Techniques de Tanger, Université Abdelmalek Essâadi,

B.P. 416 ,

Tanger, Morocco

e-mail: a_chenaoui@hotmail.com

\section{Introduction}

In metallic alloys, the mesoscopic scale involves single grains or a limited number of grains. The characteristic length of the phenomena relevant with this scale starts with a few $\mu \mathrm{m}$, which is the size of small grains in ordinary metals, and extends up to $500 \mu \mathrm{m}$. This is the size of some big grains or, which is more significant here, the size of the representative volume element as defined, for example, by Hill [1]. It is the minimum cubic volume, which can be considered homogeneous for macroscopic calculations, being large enough to average the anisotropic properties of the individual grains.

Deformation in metallic alloys is homogeneous only at its very beginning, in carefully designed mechanical tests in which the effects of friction are kept to a minimum. Then gradients appear in the displacement field and they take high values on determined locations of the material: The phenomenon is known as "localization." Although the heterogeneities at large plastic or viscoplastic deformations are multiform [2], a few considerations are a useful guide when dealing with them.

(i) In crystalline materials, the displacement of the material is accompanied by rotations of the crystal lattice that also are far from being homogeneous: Hence the study of heterogeneities must be both mechanical and crystallographic as two types of gradients have to be considered. (ii) The notion of heterogeneity is always to be associated with a specified scale. In particular, metals are microheterogeneous because of the activity of the slip systems and the microtwins, but they tend to be macrohomogeneous. A number of studies have been devoted to microscopic heterogeneities [3-5].

(iii) Some phenomena, such as shear bands, develop on different scales, growing from microscopic to macroscopic. Others are typically mesoscopic: Such are the deformation bands inside large grains or regular patterns of deformation encompassing a few small grains [6]. The scientists' attention has been attracted recently by the heterogeneity of the response at the mesoscopic level, contrasting with a smoother macroscopic behavior. This is the realm of "mesomechanics." Specific tools have been developed to measure the mechanical properties of the metals at this scale, and the corresponding values may differ from the usual macroscopic ones when determined along specific deformation paths such as low cycle fatigue loading [7]. Mesomechanics are particularly important in the study of ultrafine grained alloys [8].

(iv) A useful distinction, as found, for example, in Ref. [9], can be made between extrinsic or geometrical heterogeneities, which find their origin in the boundary conditions imposed to the test-pieces, such as necking or kink bands, and intrinsic or material heterogeneities, the sources of which have to be traced to the inner structure of the metal, irrespective of the boundary conditions. They are often analyzed as changes in the relative contribution of the many 
modes of deformation available in metals. Such are shear bands at their onset.

In this context, the purpose of this paper is to present two measurement techniques, one for the displacement field and one for the lattice rotations, which work together at the mesoscopic scale. An application is then given that puts into evidence some typical heterogeneities of the plastic deformation.

\section{Transferable Carbon Grids}

The surfaces of polished metallic samples are smooth and shiny, since test-pieces are usually polished to avoid friction and superficial defects. Hence, some marking has to be attached to them to follow their deformation. It may be a regular pattern (dots and grids) or not (speckles). Grids may be favored for several reasons. One is that their step can be adapted to the scale of the studied phenomena, ranging from the micrometer to the centimeter. Another is that they put directly in evidence the heterogeneities of deformation and allow a qualitative assessment before the measurement of the displacement field and subsequent mathematical treatment.

A well known and efficient technique is the electrolithodeposition of grids in a scanning electron microscope. It was initially designed for microelectronics, but it was applied to material science as early as 1976 [10] and has been widely used ever since $[11,6]$. The step can be any size above $2 \mu \mathrm{m}$. Nevertheless, one limitation is that the surface covered by the deposited grid is restricted by the aperture of the microscope chamber and cannot exceed a few $\mathrm{mm}^{2}$ at one go. Mesoscopic phenomena of a characteristic length of $50 \mu \mathrm{m}$ or $100 \mu \mathrm{m}$ develop on broader surfaces. A recent study performed with electrolithodeposited grids of $1 \mathrm{~mm}^{2}$ of extension [12] found that shear bands, at a certain stage of their development, extend outside the grids. It was concluded that it would have been interesting to cover the whole test-piece with one global grid.

Several technologies were considered and an attempt was made with transferable carbon grids. These are sold in sheets of a few $\mathrm{dm}^{2}$, cut at the required size with scissors and transferred on to the surface of the samples under investigation. Those used in the present study have been manufactured by a firm whose website is given in Ref. [13]. Such grids have been used for about 15 years in the field of the mechanics of composite materials [14], that is, in the range of elasticity. To the best of the authors' knowledge, it is the first time they were used on heavily deformed metals to reveal plastic heterogeneities.

Grids are created using a photoplotter. A polyester film about $0.1 \mathrm{~mm}$ thick is covered by a photographic emulsion. A laser beam approximately $10 \mu \mathrm{m}$ wide, positioned with an accuracy of $2 \mu \mathrm{m}$, impresses a grid upon it. It is then applied on to the surface of the test-piece, which has beforehand been covered with a film of glue. The sequence of the layers is thus metal/glue/grid/ polyester film. When heated, the glue polymerizes. The adhesion of the grid to the glue exceeds its adherence to the polyester film and the latter can be removed. The principles of the fabrication of such grids can be found in Ref. [15].

The complete procedure for aluminum test-pieces is described in Ref. [16]. The surface is mirror polished, the polishing ending with $\frac{1}{4} \mu \mathrm{m}$ diamond paste, and any trace of grease is removed with ethanol. The grid is also cleaned with ethanol and dried with air. A drop of cyanoacrylate gel (for example, Loctite $454 \mathrm{TM}$ ) is put at the center of the surface under investigation with a spatula. The grid is then aligned with one edge of the sample and carefully applied onto its surface, so as to chase out air bubbles. The parallelism of the bars of the grids and the edges of the sample is once more verified, and a silicon tablet is imposed on the whole so as to apply a small pressure $\left(800 \mathrm{~g}\right.$ for a surface of $\left.1 \mathrm{~cm}^{2}\right)$. It is then put in an incubator at $26^{\circ} \mathrm{C}$ during $48 \mathrm{~h}$. Subsequently, the



Fig. 1 Principle of the channel-die compression test

polyester film can be peeled off and the grid is ready. Such operations require some skill, but can be performed repeatedly in a very satisfactory manner.

In the experiments presented further below, the grids had steps of $100 \mu \mathrm{m}$ (steps down to $30 \mu \mathrm{m}$ are available). They were employed on test-pieces $10.4 \mathrm{~mm}$ high, $8 \mathrm{~mm}$ long, and $7 \mathrm{~mm}$ wide that were compressed in channel-die at room temperature at a rate of $\dot{\bar{\varepsilon}}=5 \times 10^{-3} \mathrm{~s}^{-1}$. The principle of this mechanical test, extensively used to simulate rolling conditions since 1966 [17], is recalled in Fig. 1. It consists basically of a groove in which the metal flows under the action of an indenter, so that in the case of isotropic materials it is a plane-strain test. Regardless of possible anisotropy, the deformation is measured here using the isotropic analysis. If $h_{0}$ is the initial height $(10.4 \mathrm{~mm}$ in the present case) and $h$ is the current one, the deformation of the test-piece is indicated by the compressive logarithmic strain

$$
\bar{\varepsilon}=\ln \left(\frac{h_{0}}{h}\right)
$$

The test-piece is wrapped up in Teflon ${ }^{\mathrm{TM}}$ so as to keep friction to a minimum. Large deformations are performed step by step, each corresponding to $\bar{\varepsilon}=0.15$, and the wrapping is removed at each step, allowing the observation of the grids. In the present experiments, the grids were deposited on one of the lateral sides of the test-piece $\left(10.4 \times 8 \mathrm{~mm}^{2}\right)$. This side keeps in contact with the vertical wall of the channel-die, being pressed against it by a lateral or constraint stress. The order of magnitude of the stress applied vertically by the indenter is $100 \mathrm{MPa}$. The constraint stress on the grid is a fraction of it. This fraction $\alpha$ varies with the nature of the sample. For fine-grained polycrystals, which can be considered isotropic, $\alpha=0.5$. For single crystals, it is strongly dependent on the crystallographic orientation. A theoretical analysis of single crystals having a common stable (110) orientation axis and variable elongation directions has shown that it varies from 0 to 1.5 [18]. The experimental values measured by the same authors ranged from 0 to 1 . Recently, measurements using a slightly different technique (walls of the channel-die maintained by two tightened screws instead of an adjustable jaw) have been performed on usual rolling components of texture [12]. They gave values from 0.1 to 0.8

The important fact is that the grid remains plane as the test gets on. Grids have not been tested on free surfaces, which deform in three dimensions. As will be seen below, in the case of the channel-die, the free surfaces that move through the groove become soon rugged and the grids would likely peel off or at least be of little use to assess the heterogeneities. On the contrary, on the plane vertical sides, the grids have been found in good condition up to $\bar{\varepsilon}=1.2$. This is due to the mechanical qualities of the glue, which resists intense shearing and accompanies the metal in its displacements, bearing the grid on it.

Figure 2 presents some typical situations. The test-piece is a single crystal of brass (110)[1르] orientation. Insets (a1), (a2), and (a3) of Fig. 2, presented at the same scale, show the grid first undeformed (it is then $10.4 \times 8 \mathrm{~mm}^{2}$ ) and then deformed at $\bar{\varepsilon}$ $=0.45$ and at $\bar{\varepsilon}=0.9$. For the sake of brevity, the vertical bars are simply called "bars" later on, and the horizontal ones "rungs." 


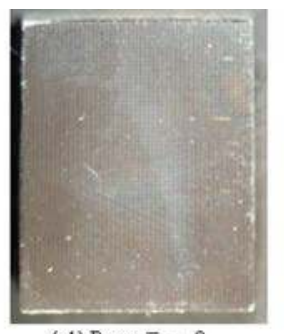

(a1) Brass $\bar{\varepsilon}=0$

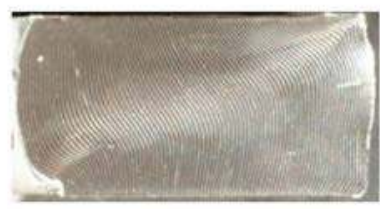

(a2) Brass $\bar{\varepsilon}=0.45$

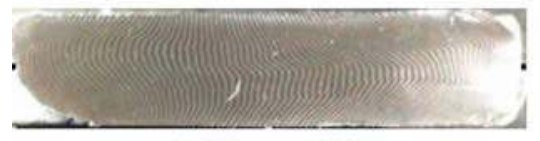

(a) Brass $\bar{z}=0.90$

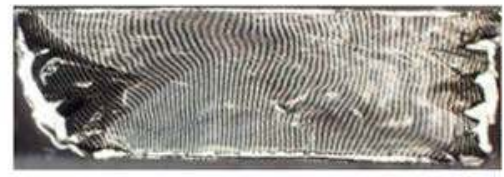

(c) Rotated Copper $\bar{\varepsilon}=0.75$

Fig. 2 Deformation of the grids and typical defects

Were the compression field is homogeneous, the bars would remain straight. That is obviously not the case. Zooms on interesting locations, magnified five times with respect to Fig. 2, insets (a1), (a2), and (a3) are presented in insets (b1), (b2), and (b3) on the same figure. In (b1), indeed, the step is exactly $0.1 \mathrm{~mm}$, but (b2) and (b3) show how the bars, which are initially about $30 \mu \mathrm{m}$ thick, become thicker while the rungs grow thinner with the deformation. This shows plainly that their own mechanical resistance is negligible and that they move with the film of glue.

However, all the grids were not perfect. Practicing the technique about 50 times yields the following considerations.

(1) The most sensitive parts of the grids are the corners and the edges in contact with the free surfaces. There is a risk of peeling off and leaving the metal bare at those locations. Comparatively, at the upper horizontal edge (indenter) and the lower one (bottom of the groove), little happens. This appears clearly in Fig. 2(c).

(2) If a scratch is made to the grid away from the edges, it evolves with the deformation of the test-piece but it does not propagate. Adhesion in the center of the test-piece is quite good.

(3) Heterogeneities entail local deformations, which are much larger than the global ones. The latter reached $\bar{\varepsilon}=1.2$ in the present experiments and, nevertheless, the grid resisted such deformations.

At the end of each step, the grids were examined with a binocular magnifier, the magnification varying from 3 to 28.5 so as to get photographs of the whole test-pieces or focus on some interesting aspect. Some light reflections were inevitable. They were taken into account in the further processing of the images.

\section{Microfocus X-Ray Texture Analysis}

Treatises on texture analysis such as Ref. [19] classify the $\mathrm{X}$-ray diffraction techniques as "macrotexture measurements." This was fully justified until most recently because the surface scanned by the incident beams was ordinarily at least $1 \mathrm{~mm}^{2}$ if information had to be gathered within a few hours. Progress in nanotechnologies leading to improved detectors and parallel progress in micromechanics allowing high precision positioning of the beams now transform X-ray diffraction into a "mesotexture measurement." The necessary outfit is currently referred as "microfocus." The irradiated spot, basically a disk that becomes ellipsoidal when the sample is tilted and rotated, may have a diameter as small as $10 \mu \mathrm{m}$.

The equipment for texture analysis consists of an X-ray tube, a goniometer, a detector, and subsequent signal processing. The second and the fourth elements of this sequence are much the same in macro- and mesodevices. Nevertheless, in the latter case, a sample holder with three degrees of freedom and excellent positioning is required. The first and the third elements present specificities, which are outlined below.

Because of the positioning requirements, the X-ray source must be contained in a very rigid tube (made of ceramics, for instance). It emits through an X-ray lens, which sends the photons through glass fibers. This acts as a wave guide, so that the beam is almost perfectly parallel to the axis of the tube: The angular discrepancy may be smaller than $0.1 \mathrm{deg}$. The distance between the source and the goniometer is the same as in the macroscopic case (the usual $250 \mathrm{~mm}$, for example). The diameter of the incident beam ranges from $10 \mu \mathrm{m}$ to $100 \mu \mathrm{m}$ depending on the needed resolution. The detector consists of a receiving slit and a counter that is more sophisticated than in conventional applications, since it performs a linear multichannel detection. The integration range can be adjusted to the spread of the diffraction peak, which is useful when the spread grows with deformation.

The results presented below were obtained with $100 \mu \mathrm{m}$ foci. Specifications on the device can be found on the manufacturer's website [20]. When fitted on a conventional equipment, the mounting and dismounting of the spare parts specific to mesoscopic measurements (source, detector, etc.) take less than $1 \mathrm{~h}$. Since aluminum was investigated, copper was used as the anode material, the characteristic line being $K \alpha$ (wavelength of 0.15418 $\mathrm{nm})$. In the X-ray tube, the voltage was $45 \mathrm{kV}$ and the intensity of the current was $40 \mathrm{~mA}$. About $3 \times 10^{5}$ photons/s were emitted, out of which a few thousand reached the counter. Their exact number depends on the crystal orientation and on the deformation. The tilt angle $\psi$ was varied $0<\psi<85 \mathrm{deg}$ by steps of $1 \mathrm{deg}$. The same step was taken for the rotation angle $\varphi$. Integration time was set to $5 \mathrm{~s}$, so that one pole figure was obtained within $2 \mathrm{~h}$. Four pole figures with different diffraction planes $\{h k l\}$ were drawn to calculate the orientation distribution functions (ODFs).

As regards the heterogeneities of deformation, the salient features of microfocus X-ray investigation are as follows.

(1) It works at typically mesoscopic scales. Not only does the diameter of the beam range from $10 \mu \mathrm{m}$ to $100 \mu \mathrm{m}$, but the penetration depth too is of the same order of magnitude. For example with aluminum, most of the reflected intensity comes from a layer situated between $50 \mu \mathrm{m}$ and $150 \mu \mathrm{m}$ below the surface of the sample, with variations due to the $\{h k l\}$ poles and the tilt.

(2) The presence of a fiducial grid has no interference with the signal. It is obvious with carbon grids. With gold or platinum grids half a micron thick, it would be much the same.

(3) Due to the energy of the X-rays, it works on all types of metals, including the light ones, whatever the density of dislocations. This point is crucial with heavily cold deformed metals.

A comparison comes to mind with the techniques of orientation 
measurements based on the Kikuchi diffraction, especially the electron backscattered diffraction (EBSD) method. The latter renders outstanding services nowadays: See, for example, Ref. [21], especially because the time necessary for one measurement is in the order of milliseconds. The microfoci, in contrast, are time consuming. The two techniques, in fact, have different fields of interest. The EBSD instruments are currently designed for microscopic investigation. Were they applied to the mesoscopic range, the following problems would arise.

(1) The layer that gives the signal is about $1 \mu \mathrm{m}$ thick just below the surface, which yields no proper information at the mesoscopic scale if several strata of grains are to be found in a depth of, say, $100 \mu \mathrm{m}$.

(2) If the dislocation density rises sharply, the quality index falls, especially with light metals. When assessing the texture of the mesoscopic layer, considering only the favorably oriented grains would be misleading.

(3) Even in plane-strain compression, heavy deformation produces ripples on the surface. EBSD requires smooth surfaces, with no peaks and valleys of more than $1 \mu \mathrm{m}$. The experiments described in Ref. [6] showed that beyond $\bar{\varepsilon}$ $=0.3$ aluminum samples compressed in channel-die needed repolishing, which consequently removed the surface grid and prevented further investigation of the displacement field.

These considerations prompted the authors to search for alternative tools in the case of cold-worked metals.

\section{Heterogeneities of Deformation Revealed by the Carbon Grids}

Much work has been done on the heterogeneities induced by rolling or channel-die compression of metals, particularly at the microscopic scale. The microscopic evolution has been investigated in polycrystals [21] and in single crystals $[17,22,23]$. The latter put in evidence the importance of the orientation of the crystal lattice with respect to the elements of symmetry of the channel-die, especially the $\left(X_{1}, X_{3}\right)$ plane. For the mesoscopic study, an aluminum alloy with $1 \%$ by weight of magnesium and practically no other elements was chosen. It offers better mechanical resistance than pure aluminum. A homogenization annealing at $625^{\circ} \mathrm{C}$ during $48 \mathrm{~h}$ followed by air quenching ensures that there are no $\mathrm{Al}_{6} \mathrm{Mn}$ precipitates, at least larger than $0.05 \mu \mathrm{m}$. Indeed, it is remarkably homogeneous before deformation and no segregations are noted. Hence the reproducibility of the results are documented below. More than 50 tests in all were performed on polycrystals with grains of about $20 \mu \mathrm{m}$ and on single crystals of different crystallographic orientations. For test-pieces with the same characteristics, the results were the same.

As far as kinematics is concerned, a reference deformation is given by the homogeneous analytical model. This relies on rateinsensitive crystal mechanics and assumes that there are neither imperfections in the metal nor friction against the channel-die (no intrinsic or extrinsic causes of heterogeneities). Hence the results are readily computed [24]. They provide an element of comparison with the nonhomogeneous experimental results.

According to the homogeneous analytical model, the channeldie compression produces plane strain on isotropic materials. Such is the case of the polycrystals of the present study, which exhibit small grains and almost no crystallographic texture. It is also true when the plane of the channel-die is a plane of symmetry for the compressed single crystals, viz., a $\{100\}$ or $\{110\}$ plane in fcc lattices. Hence, in Table 1, the orientations of the single crystals are listed according to this criterion. First come the ones with three planes of symmetry common with the channel-die. They are only four orientations, cube, $\mathrm{U}$, goss, and cube rotated $\pi / 4$ around
Table 1 List of tested crystallographic orientations

\begin{tabular}{lccc}
\hline \hline Orientations & $\begin{array}{c}\text { Miller } \\
\text { indices }\end{array}$ & $\begin{array}{c}\text { Plane of the } \\
\text { channel-die }\end{array}$ & $\begin{array}{c}\text { Axes of material } \\
\text { rotation }\end{array}$ \\
\hline Cube $(\mathrm{Cu})$ & $\{001\}<100\rangle$ & $\{010\}$ & - \\
$\mathrm{U}$ & $\{110\}\langle 1 \overline{1} 0\rangle$ & $\{00 \overline{1}\}$ & - \\
Goss & $\{110\}\langle 001\rangle$ & $\{1 \overline{1} 0\}$ & - \\
Rotated cube & $\{001\}<110\rangle$ & $\{1 \overline{1} 0\}$ & - \\
Copper $(\mathrm{C})$ & $\{112\}\langle 11 \overline{1}\rangle$ & $\{1 \overline{1} 0\}$ & $X_{2}$ \\
Rotated copper & $\{11 \overline{1}\}<112\rangle$ & $\{1 \overline{1} 0\}$ & $X_{2}$ \\
Brass $(\mathrm{Bs})$ & $\{110\}\langle 1 \overline{1} 2\rangle$ & $\{1 \overline{1} \overline{1}\}$ & $X_{3}$ \\
Strange $(\mathrm{S})$ & $\{\overline{1} 23\}\langle 12 \overline{1}\rangle$ & $\{4 \overline{1} \overline{2}\}$ & $X_{1}, X_{2}$, and $X_{3}$ \\
\hline \hline
\end{tabular}

the $X_{3}$ axis. Then come the copper and rotated copper orientations, for which the only common element of symmetry is the plane of the channel-die.

Plane strain conditions are not fulfilled for other single crystals. With the brass orientation, the compression axis $\left(X_{3}\right)$ is initially a (110) crystallographic axis and remains such during all the compression. That is why the grids deposited on the lateral face show no or little plastic shear. Meanwhile, the material lines initially in the transverse direction $X_{2}$ rotate around $X_{3}$ as the crystal plastically shears in the $\left(X_{1}, X_{2}\right)$ plane, as studied experimentally and theoretically in Ref. [25]. The strange orientation, on the contrary, is an example of the ordinary orientations, which have no element of symmetry in common with the channel-die.

However, grids only attest of the deformation of the layer of material in contact with the wall of the channel-die and this layer undergoes plane deformation. The deformation of the test-piece, on the contrary, is described by a three dimensional tensor. A rigorous analysis of the homogeneous deformation of a sample in channel-die compression is given in the Lagrangian frame in Ref. [26]. The various tensors used in the spatial or Eulerian $\left(x_{1}, x_{2}, x_{3}\right)$ frame can be deducted from it. Among them is the gradient of the displacement-rate $\mathbf{L}$ defined by

$$
\mathbf{L}=\operatorname{grad}(\dot{\mathbf{u}})=\left[\frac{\partial \dot{u}_{i}}{\partial x_{j}}\right]
$$

u being the velocity field in the test-piece. Since the elastic deformation is small with respect to the plastic one, the volume does not change and $L_{i i}=0$.

The boundary conditions are shown as follows.

(1) The channel-die has a fixed width. This implies $L_{22}=0$. Therefore $L_{11}=-L_{33}$, the latter being imposed by the indenter $\left(L_{33}=5 \times 10^{-3} \mathrm{~s}^{-1}\right.$ in the present experiments). The area covered by the grid remains constant.

(2) Since the bottom of the groove and the indenter remain horizontal throughout the compression, the material lines, when initially horizontal, remain such throughout the deformation. Hence $L_{31}=0$.

(3) Because of the walls of the channel-die, the initially vertical material lines remain upright, so that $L_{21}=0$.

(4) Shear is constrained in the $\left(X_{2}, X_{3}\right)$ plane, so that $L_{23}=L_{32}$ $=0$.

Therefore the gradient of the displacement-rate can be written as

$$
\mathbf{L}=\left[\begin{array}{ccc}
-L_{33} & L_{12} & L_{13} \\
0 & 0 & 0 \\
0 & 0 & L_{33}
\end{array}\right]
$$

The grids deposited on the lateral side give information only on the components $L_{11}, L_{13}, L_{31}$, and $L_{33}$. The examination of the images of the grid at two successive steps (separated by $\Delta \bar{\varepsilon}$ 


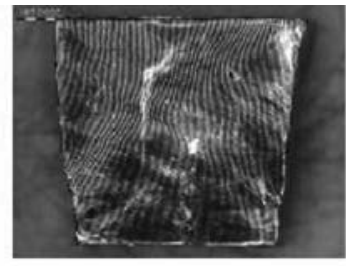

(a) $\bar{\varepsilon}=0.15$

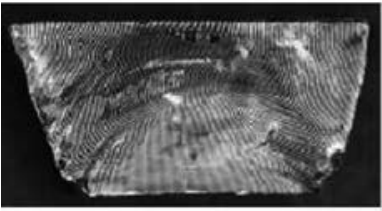

(c) $\bar{\varepsilon}=0.45$

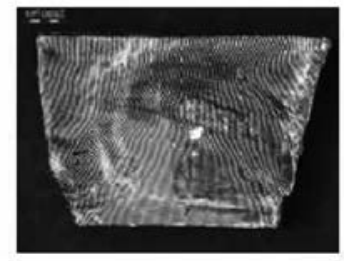

(b) $\bar{\varepsilon}=0.30$

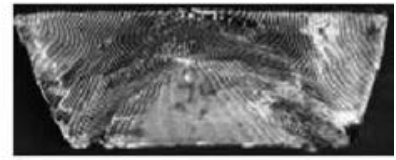

(d) $\bar{\varepsilon}=0.60$

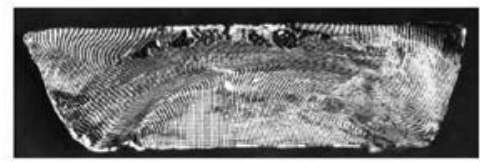

(e) $\bar{\varepsilon}=0.75$

Fig. 3 Sequence of deformation of a polycrystalline sample

$=0.15$ ) gives access to these components in real deformation. Here, the homogeneous analysis proves to be a useful guide. Throughout the grid we observe the following.

(1) $L_{33}$ (and consequently $L_{11}$ ) differ little from the value imposed by the indenter and its variations over the investigated surface are low.

(2) The inclination of the rungs with respect to horizontal is small (as a rule less than $5 \mathrm{deg}$ ), so that $L_{31}$ is small or zero.

(3) The most salient feature of the deformation is the shear represented by $L_{13}$, which can be very large and have dramatic variations across the grid. It is visualized by the slant of the bars, which in some cases rotate 90 deg or more.

No quantitative analysis of the deformation is offered here. Such work is in progress, using image analysis techniques currently adapted from the "grid method" [27]. This will be the subject of specific future papers. This paper only deals with the qualitative examination of the grids, which prompts the thought that the heterogeneities of deformation preferably take the form of shears. Nevertheless, this statement must be understood keeping in mind the following.

(1) In plane deformation with a constant area, any field of displacement may be analyzed as a shear. It suffices to take as a reference frame the axes rotated $\pi / 4$ from the principal axes. So it must be stressed that the deformation is presently evaluated in the axes of the compression, in which there is a mixture of shortening, elongation, and shear.

(2) The sheared zone often forms ribbons or bands at the surface of the samples, but this does not mean that it is automatically a "shear band" in the sense given in classifications such as Ref. [2]. The term shear bands is used here for specific metallurgical phenomena, originating as microbands inside the test-piece and developing on larger scales $[3,28,29]$. They are characterized by strong crystallographic rotations. Most of the observed ribbons are simply "deformation bands," the frontiers between them being sometimes called "transition bands."

The sequence of the deformation of polycrystals is shown in Fig. 3. The symmetry of the figure with respect to the vertical axis is globally retained throughout the deformation. Signs of heterogeneity appear as early as $\bar{\varepsilon}=0.15$. As often pointed out in

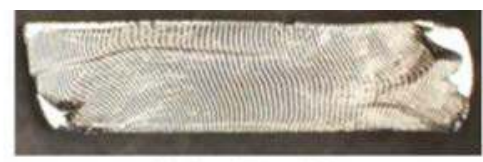

(a) Goss $\bar{\varepsilon}=0.90$

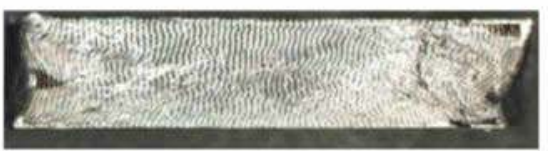

(b) Cube $\bar{\varepsilon}=0.90$

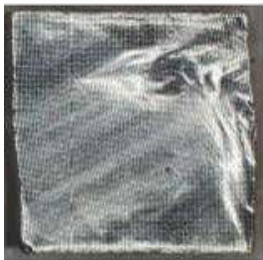

(c1) Copper $\bar{\varepsilon}=0.15$

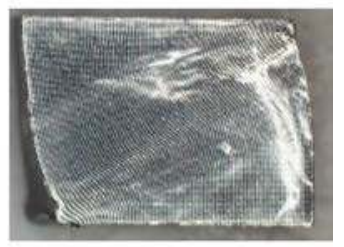

(c2) Copper $\bar{\varepsilon}=0.30$

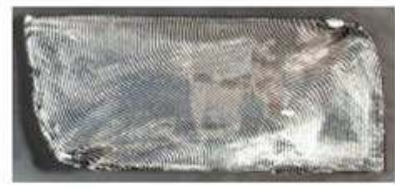

(c3) Copper $\bar{\varepsilon}=0.60$

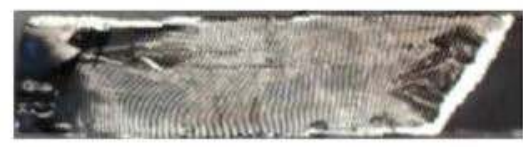

(d) Strange $\bar{\varepsilon}=0.75$

Fig. 4 Deformation of remarkable single crystals

channel-die studies, the upper part of the sample, in contact with the indenter, becomes wider than the bottom one. This has been explained by finite element simulations taking into account friction [30] and is due to the fact that beneath the indenter, the velocity is imposed by the testing machine while it is zero at the bottom of the groove. The sensitivity of the corners of the testpiece comes from the fact that on their horizontal side, they are submitted to friction, while they are friction free on the vertical one. In some cases, the lower parts of the free surfaces remain approximately vertical up to $\bar{\varepsilon}=0.30$, then yield and crush against the bottom of the groove: See the right corner of Fig. 3(b), for example. This of course triggers heterogeneities that are clearly extrinsic. They take the form of an arc-shaped band in which the shear is large. It absorbs much of the deformation and its boundaries with the rest of the matrix are well delimited. In the matrix, the undulations of the bars have characteristic lengths of several millimeters. It can be said that the deformation of the matrix remains "mesohomogeneous," in spite of the gradients occurring in the band.

Figure 4 shows a few situations typical of single crystal deformation. Goss is considered as the most "stable" of all orientations [23], which means that the homogeneous analytical model predicts neither change in shape nor crystal rotation. Only some barreling can be seen at $\bar{\varepsilon}=0.90$ in Fig. $4(a)$. The latter shows some tearing of the grid at the edges of the sample, as an example of the shortcomings of the transferable carbon grid technology. The rungs remain almost horizontal and the bars have large undulations, characteristic of a mesohomogeneous deformation.

Although they possess the symmetries of the channel-die, the cube, $\mathrm{U}$, and rotated cube (more precisely: cube rotated $\pi / 4$ 
around $X_{3}$ ) orientations have a quite different behavior and their split into regions of contrasting crystallographic evolutions has been widely accounted for in literature [31]. Figure 4(b) shows how it appears at the mesoscopic scale. The bars of the cube sample are jarred and exhibit serrations with a characteristic length of a few hundred microns, so that the orientation can be classified as mesoheterogeneous. The corresponding evolution of the crystal lattice is investigated below with the help of the microfoci.

Copper is one of the most studied crystal orientations [32] because of its well understood slip geometry. Four active slip systems, two coplanar and two codirectional, are simultaneously active in channel-die compression and produce a plane $\left(X_{1}, X_{3}\right)$ deformation, as can be seen, in particular, in Fig. 1 of Ref. [33]. For metals with low stacking fault energy, or for metals with a higher one deformed at cryogenic temperatures [34], channel-die compression produces a large shear band containing the transverse direction. The homogeneous analytical model assuming uniform stress and unrestricted longitudinal shear (the so-called "Tucker conditions" [31]) predicts the plane deformation, the lateral face of the test-piece transforming into a parallelogram, without rotation of the crystal lattice.

The evolution of the microgrids presented in Figs. 4 and 7 confirms these observations. The free surfaces curve inwards with a global slant, as predicted. A large deformation band around the shortest diagonal of the lateral face gradually occupies most of the test-piece. The conventional X-ray measurements [12] show the stability of the orientation, the scatter growing slowly around the initial positions without discernible split until $\bar{\varepsilon}>1$.

Step by step analysis of the compression yields the following remarks.

(1) At $\bar{\varepsilon}=0.15$ (Fig. 4 (c1)) the deformation is still rather homogeneous and the longitudinal shear is small. Nevertheless, bands inclined $30 \mathrm{deg}$ with respect to the horizontal become visible.

(2) At $\bar{\varepsilon}=0.30$ (Fig. 4 (c2)), the geometry of the deformation becomes apparent. Two triangles, one beneath the indenter, the other in contact with the bottom of the groove, and both with one side on a free face, are relatively unaffected by the shear. The latter concentrates in the band-shaped central zone. It is noteworthy that at $\bar{\varepsilon}=0.30$, it is inclined about 20 deg with respect to the horizontal: That is precisely the inclination of the plane of the coplanar slip systems, which cause most of the deformation since the glide on them is twice the glide on the codirectional ones [33].

(3) The phenomenon grows in intensity when the free surfaces begin piling up, the left one against the bottom of the groove and the right one against the indenter. When the shorter diagonal is itself inclined $35 \mathrm{deg}$, that is around $\bar{\varepsilon}$ $=0.45$ (Fig. 7 (a1)), it is at its strongest. Closer examination shows that the central zone consists of a stacking of three deformation bands within which the shear is approximately constant, separated at places by sharp transition zones. The longitudinal shear is the highest in the middle band. Measured from the inclination of the initially vertical bars, it is of the order of 2 (tilt of about $60 \mathrm{deg}$ of the bars with respect to the vertical).

(4) When processed further, geometry changes (Fig. 4 (c3)). Beneath the indenter and at the bottom of the groove, the slant of the bars reveals the friction exerted on the testpiece; but its periphery, especially where it borders on the free surfaces, is less affected by the shear than the inner part. This has rather well delineated boundaries and is characterized by increasing longitudinal shears. At $\bar{\varepsilon}=0.90$ (Fig. 7 (b1)), the latter may be such that at places the initially vertical bars overrun the horizontal and are found in opposition with the general movement of the deformation.
The copper case illustrates the mixed character of the heterogeneity of the deformation. It has definitely extrinsic causes because it is triggered by concentrations of stress at the corners of the sample and is influenced by the friction exerted by the indenter and the groove. But the slope of the deformation bands can only be explained by crystallographic considerations. The conclusion would have been the same if the test-piece, deformed in liquid nitrogen, had developed shear bands with a characteristic $35 \mathrm{deg}$ inclination to the horizontal, as it does for a wide range of texture orientations: See, for example, Ref. [35].

Examples of the brass orientation have already been shown in Fig. 2. In the $\left(X_{1}, X_{3}\right)$ plane there is no overall finite shearing of the crystal but local material rotations take place around the $X_{2}$ axis, as can be seen from the shadow of the free rotating surfaces, visible on the photographs. The strange orientation rotates strongly around the $X_{2}$ and $X_{3}$ axes: See Fig. $4(d)$, but no serrated bars can be seen on it, so that its deformation is definitely mesohomogeneous. Other orientations, chosen among the single crystals available in the laboratory, showed the same behavior as strange. So it appears that the mesoheterogeneity is characteristic of a few highly symmetrical orientations, the most important being cube [36].

In order to sum up the above orientations, it must be stressed that the channel-die device, with free surfaces pushed through a gauge when the indenter moves down, triggers heterogeneities of deformation of its own. These are clearly visible at the mesoscopic scale. This by no means lessens the interest of the test, which is very much representative of the rolling process, especially from the point of view of the evolution of textures.

\section{Microfocus X-Ray Analysis}

Numerous examples of measurements by conventional X-ray equipment are available in literature, especially analyses of the rotations of single crystals in channel-die compression [23]. Some crystallographic orientations, for example, are known as stable, which means that they do not rotate with the deformation. The others are labeled "unstable." For most of them, the whole sample keeps a global orientation, the spread around it increasing slowly with the deformation; but for some like cube, the sample splits into zones rotating differently [37].

The work performed here with microfocus X-rays was not aimed at verifying systematically well established results. Rather, it was assigned two specific goals. The first was to collect information on the way some unstable orientations split. The second was to assess the respective role played by the material rotation and the crystallographic rotation in typically heterogeneous situations.

As explained in Sec. 2, the examination of the grid gives access to four components of the gradient of displacement-rate and hence to the component $\Omega_{13}$ of its skew-symmetric part $\boldsymbol{\Omega}=\left(\mathbf{L}-\mathbf{L}^{T}\right) / 2$. $\boldsymbol{\Omega}$ is the material rate of rotation tensor. Since the grid remains plane, only its component $\Omega_{13}$ is of interest. Calculating $\Omega_{13}$ in the rigorous formalism of large deformations requires care, but in the present qualitative phase of the work and due to the fact that the rungs have little inclination, it suffices to consider the slant of the bars (measured in radians) and divide by two to have a rough assessment of its magnitude. The lattice rotation-rate tensor or lattice spin, on the other side, is detected in the pole figures. Its complete evaluation involves three components $R_{12}, R_{13}$, and $R_{23}$. Unlike the grid, it is not confined to a plane by the walls of the channel-die. Here, only $R_{13}$ can be compared with the material rotation-rate. This component is given by examining the rotation of the diffraction peaks on any pole figure, provided that this has been drawn on the plane $\left(X_{1}, X_{3}\right)$. Such is the case in the pole figures presented below. The reader will note that they differ from the usual ones, obtained on the $\left(X_{1}, X_{2}\right)$ plane for rolled sheets. That is why in this paper, the Miller indices of the $\left(X_{1}, X_{3}\right)$ plane have been mentioned in the pole figures of single crystals. 


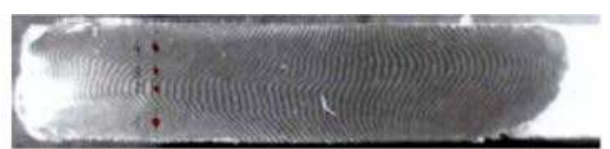

(a) Brass $\bar{\varepsilon}=0.90$

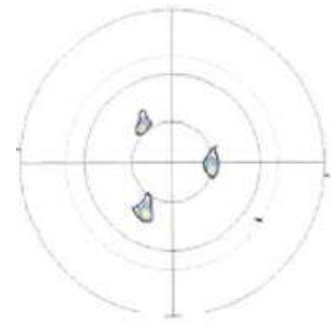

Point 2

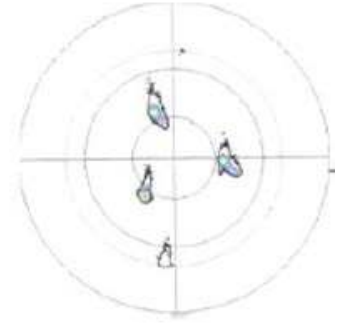

Point 4 (b) Poles $\{110\}$, projection plane $\{1 \overline{11}\}, \bar{\varepsilon}=0.90$

Fig. 5 Microfocus X-rays on the brass orientation

In accordance with the objectives set above and on the basis of previous data, samples of three different orientations were chosen for microfocus $\mathrm{X}$-ray investigation:

(1) brass, without overall material rotation or lattice rotation on the lateral face

(2) U, also without overall material rotation but showing crystallographic instability, even with conventional X-rays

(3) copper, with overall material rotation but no macroscopic lattice rotation

Contrasted results concerning the split in crystallographic orientations were put in evidence by directing the X-ray beam on various spots on the surface of brass and $U$ samples. In the case of brass, the characteristic positions of the poles, which appear in Fig. 5(b), were found to be much the same throughout the side of the sample at its different stages of deformation. At $\bar{\varepsilon}=0.90$, the diffraction spots found at point 2 spread exactly around the poles of a perfect brass orientation. The main discrepancy with this ideal orientation can be seen at point 4 and it consists of a rotation of about $15 \mathrm{deg}$. On the contrary, differing results were obtained at points $A, B$, and $C$ on the $\mathrm{U}$ sample, as shown in Fig. $6(b)$. The scatter in the crystallographic orientations was large and reached $45 \mathrm{deg}$ at $\bar{\varepsilon}=0.60$. Besides, the pole figures at point $B$ revealed a split of orientations on each side of the $X_{2}$ axis. One of the components was largely dominant at point $A$, the other at point $C$. So

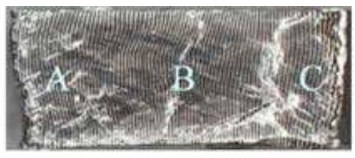

(a) $\mathrm{U} \bar{\varepsilon}=0.60$

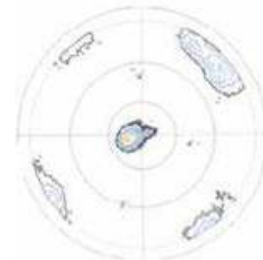

Point A

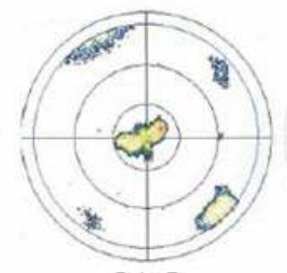

Point B

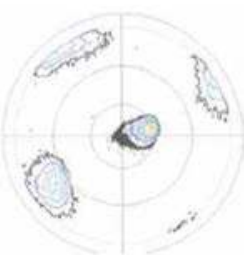

Point C (b) Poles $\{100\}$, projection plane $\{00 \overline{1}\}, \bar{\varepsilon}=0.60$

Fig. 6 Microfocus $\mathrm{X}$-rays on the $\mathrm{U}$ orientation

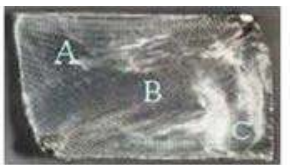

(a1) Copper $\bar{\varepsilon}=0.45$

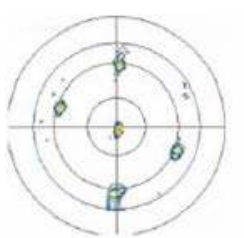

Point A

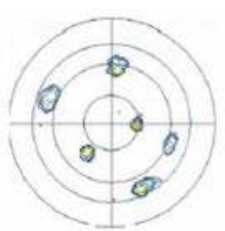

Point B

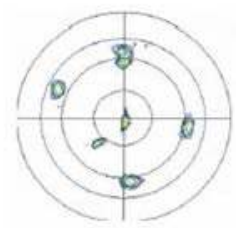

Point C (a2) Poles $\{110\}$, projection plane $\{1 \overline{1} 0\}, \bar{\varepsilon}=0.45$

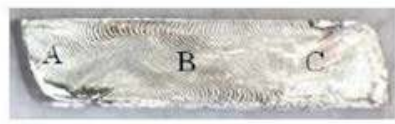

(b1) Copper $\bar{E}=0.90$

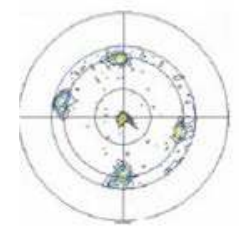

Point A

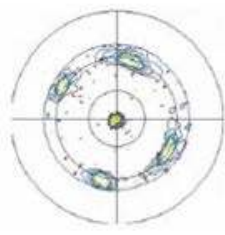

Point B

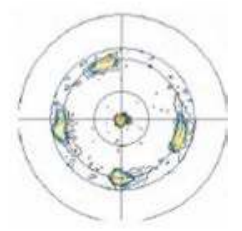

Point C (b2) Poles $\{110\}$, projection plane $\{1 \overline{1} 0\}, \bar{\varepsilon}=0.90$

Fig. 7 Microfocus X-rays on the copper orientation

the test-piece progressively splits into regions of different crystallographic orientations. Meanwhile, as documented in Ref. [17], no macroscopic deformation band was put in evidence by the grid.

Putting in evidence the difference between the material and the crystallographic rotations supposes measuring the texture at various steps of deformation while assessing the rotation of the grid. Here the microfocus X-rays have a decisive advantage. As shown in Fig. 5(a), they were directed on four spots aligned along a vertical crossing with contrasting levels of longitudinal shear. The latter is almost 0 at point 2 while it is about 1 at point 4 . In spite of this, there is no great change in the crystallographic orientations and along the vertical line, the pole figures show smooth oscillations, far from the sharp gradients of the field of material rotation-rates. The plastic spin $\boldsymbol{\omega}$, calculated as the rate difference $\boldsymbol{\omega}=\boldsymbol{\Omega}-\mathbf{R}$, is due to the glides of the slip systems that produce rotations within the axes of the crystal lattice. These glides clearly appear as the key factor in the localization of the deformation.

Figure 7 allowed to gather further information on the copper case. At $\bar{\varepsilon}=0.45$, the microfocused beams were pointed at three spots:

(1) point $A$, in the upper, unsheared triangle, close to the punch

(2) point $B$, in the zone of the bands, at a spot were there is an inflection in the slant of the vertical bars of the grid (referred above as transition band)

(3) point $C$, in the lower, unsheared triangle, close to the bottom of the groove

Point $A$ exhibits an almost perfect copper texture (Fig. 7 (a2)). It is nearly the case with point $C$, but the bottom left quadrant of the pole figure presents a spot that does not belong to the copper orientation but to the component displayed at point $B$. Here, the 
copper component has disappeared to be replaced with a component whose Miller indices are not classical but approximately $\left\{\begin{array}{lll}1 & 0 & 2\end{array}\right\}\left\langle\begin{array}{lll}10 & 6 & \overline{5}\end{array}\right\rangle$.

This shows clearly that the development of the deformation bands in the copper texture is accompanied by lattice rotations that, contrarily to what was seen above, do not take place around axis $X_{2}$. Between the initial $\left\{\begin{array}{lll}1 & 1 & 2\end{array}\right\}\left\langle\begin{array}{lll}1 & 1 & \overline{1}\rangle\end{array}\right)$ and $\left\{\begin{array}{lll}1 & 0 & 2\end{array}\right\}$ $\times\left\langle\begin{array}{lll}10 & 6 & 5\end{array}\right\rangle$, calculations based on the Mackenzie formula (see, for example, Ref. [38]) show a rotation of $26 \mathrm{deg}$ around an axis close to $\left\langle\begin{array}{lll}\overline{9} & 6 & 5\end{array}\right\rangle$.

Concerning point $A$, it must be specified that the technology of the microfoci may explain the coexistence at this point of the copper texture and of an element of $\left\{\begin{array}{lll}1 & 0 & 2\end{array}\right\}\left\langle\begin{array}{lll}10 & 6 & 5\end{array}\right)$. When the sample is tilted and rotated, the impact of the beam becomes ellipsoidal and its major axis reaches $500 \mu \mathrm{m}$ or more. That is why some zone influenced by the deformation bands may have been reached while the other diffraction peaks in the pole figure were unmistakably copper.

At $\bar{\varepsilon}=0.90$, the microfoci were also pointed at three spots $A, B$, and $C$ (Fig. 7 (b1)) but the results were less contrasted. Although the slant of the vertical bars revealed a wide range of material rotations, all the microfocus X-ray analyses gave results of the copper type, with some local lattice rotations around the $X_{2}$ axis and a scattering increasing with the lateral shear. At point $A$ there was little rotation, at point $B$ the rotation was 15 deg (counterclockwise), and it was again $15 \mathrm{deg}$ (clockwise, this time) at point $C$. The $\left\{\begin{array}{lll}1 & 0 & 2\end{array}\right\}\left\langle\begin{array}{lll}10 & 6 & 5\end{array}\right\rangle$ orientation had completely disappeared. For the authors, such a return to the copper orientation was unexpected and previous conventional X-ray measurements in their laboratory had given no hint of it [12].

The microfoci were able to put in evidence in a straightforward manner the crystallographic rotations that accompany the localization of the deformation. They also confirmed that the brunt of the material rotation in the metallic alloys relies on the glide of the slip systems and not on the rotation of the crystal lattice.

\section{Conclusions}

The work presented above calls for two sorts of conclusive remarks, some centered on the capabilities of the techniques employed and others on the plastic deformation of metals in channeldie.

The transferable carbon grids proved an efficient technique to measure the heterogeneities of deformation in channel-die compression. They could be used up to large plastic deformations $(\bar{\varepsilon}$ $>1.0$ ). In this paper only a qualitative assessment has been performed but the grids could also be used for quantitative extensometry, all the more because they remained plane. They may tear off at the corners of the test-pieces and on the edges of the free surfaces. Nevertheless, a few tests of uniaxial compression between two platens have been done in which the carbon grids proved satisfactory too. A broader assessment of their capabilities would imply their use with a variety of mechanical tests, each of them having its own constraints. Rugged surfaces and jagged edges have definitely to be feared.

Transferable carbon grids are not the only available technique to measure the plastic deformation of metals at the mesoscopic scale. It should be possible to adapt the electrolithodeposition of golden and platinum grids to cover continuously surfaces of a few $\mathrm{cm}^{2}$. Metal grids can work at high temperatures. Laser should also provide efficient markings. With laser, the case of single crystals is somewhat special because of the risks of local melting. But femtosecond lasers could be the solution to mark them with a regular pattern of dots.

Microfocus X-rays definitely offer all the qualities that make $\mathrm{X}$-rays such a useful technique. They can measure crystal orientation on rugged surfaces, however, heavy the deformation, with- out wiping off the grid through polishing. Their limitation arises from the time required for the measurements, about four times what is needed with a conventional X-ray device.

While the techniques offered new perspectives, the plastic deformation in the channel-die was a classical topic and the lessons of the experiments were confirmations of previous observations. Nevertheless, some points have appeared with peculiar clarity.

The grids show that lateral shears are present very soon in the compression in channel-die. The width of the bands and their rate of evolution depend on the crystal orientations. Further quantitative analysis should be able to classify the latter with respect to their propensity to shearing. The observation of the grids also emphasizes that few crystal orientations (the highly symmetric ones, cube, $U$, and rotated cube) offer widespread mesoheterogeneities, the latter being much more concentrated in the other orientations.

The specific heterogeneities of deformation due to the channeldie were also brought out, along with the competition between the intrinsic and extrinsic causes of the phenomenon. It was also confirmed that the crystalline rotation has little correlation with the material rotation, the former being far less affected by intense gradients than the latter, at least at the mesoscopic scale.

\section{Acknowledgment}

The authors are most grateful to all who helped in the laboratory at the Mining School of Saint-Etienne, especially E. Garrigou. The task benefited from the financial support of the FrancoMoroccan Volubilis program, Integrated Action No. MA/99/04.

\section{References}

[1] Hill, R., 1963, "Elastic Properties of Reinforced Solids: Some Theoretical Principles," J. Mech. Phys. Solids, 11, pp. 357-372.

[2] Gorczyca, S., Dymek, S., Ryś, J., Maslanka, M., and Wróbel, M., 1986, "Nomenclature for Structural Heretogeneities Produced by the Deformation of Metals," Arch. Metall., 31, pp. 23-32.

[3] Korbel, A., and Martin, P., 1986, "Microscopic Versus Macroscopic Aspect of Shear Bands Deformation," Acta Metall., 34(10), pp. 1905-1909.

[4] Godfrey, A., Juul Jensen, D., and Hansen, N., 1998, "Slip Pattern, Microstructure and Local Cystallography in an Aluminium Single Crystal of Brass Orientation $\{110\}\langle 112\rangle, "$ Acta Mater., 46(3), pp. 823-833.

[5] Paul, H., Driver, J. H., Maurice, C., and Jasieński, Z., 2002, "Crystallographic Aspects of the Early Stages of Recrystallisation in Brass-Type Shear Bands,' Acta Mater., 50, pp. 4339-4355.

[6] Chapelle, D., and Darrieulat, M., 2003, "The Occurrence of Shear Banding in a Millimeter Scale (153)[634] Grain of an Al-4.5\% Mg Alloy During Plane Strain Compression," Mater. Sci. Eng., A, 347, pp. 32-41.

[7] Ye, D., Matsuoka, S., and Nagashima, N., 2007, "Determination of Fatigue Mesoscopic Mechanical Properties of an Austenitic Stainless Steel Using Depth-Sensing Indentation (DSI) Technique,” Mater. Sci. Eng., A, 456(1-2), pp. $120-129$.

[8] Tyumentsev, A. N., Pinzhin, Yu. P., Tretjak, M. V., Korotaev, A. D., Ditenberg, I. A., Valiev, R. Z., Islamgaliev, R. K., and Korznikov, A. V., 2001, "Evolution of Defect Substructure of Metal Alloys at Microscopic and Mesoscopic Level Under Torsion," Theor. Appl. Fract. Mech., 35(2), pp. 155-161.

[9] Needleman, A., 1988, "Continuum Mechanics Studies of Plastic Instabilities," Rev. Phys. Appl., 23, pp. 585-593.

[10] Attwood, D. G., and Hazzledine, P. M., 1976, "A Fiducial Grid for HighResolution Metallography,” Metallography, 9, pp. 483-500.

[11] Karimi, A., 1984, "Plastic Flow Study Using the Microgrid Technique," Mater. Sci. Eng., 63, pp. 267-276.

[12] Poussardin, J. Y., 2003, "Caractérisation et évolution des bandes de cisaillement dans les monocristaux d'Al-Mn de différentes orientations cristallographiques," Ph.D. thesis, Ecole des Mines de Saint-Etienne, France.

[13] www.laser-techno.com

[14] Mathias, J. D., Balandraud, X., and Grédiac, M., 2006, "Experimental Investigation of Composite Patches With a Full-Field Measurement Method," Composites, Part A, 37, pp. 177-190.

[15] Piro, J. L., and Grédiac, M., 2003, "Sur une méthode d'obtention de grilles transférables destinées à la mesure optique de champs de déplacement," Proceedings of the Congress Held by the French Society for Optics, Belfort, France, Nov., P. Smigielski, ed., pp. 491-495.

[16] Zhani, K., 2006, "Protocole de dépôt des grilles transférables préencollées," Ecole des Mines de Saint-Etienne, Internal Report No. 2006-34.

[17] Chin, G. Y., Nesbitt, A. J., and William, A. J., 1966, "Anisotropy of Strength in Single Crystals Under Plane Strain Compression," Acta Metall., 14, pp. 467-476.

[18] Wonsiewicz, B. C., Chin, G. Y., and Hart, R. R., 1971, "Lateral Constraints in 
Plane Strain Compression of Single Crystals," Metall. Trans., 2, pp. 2093 2096.

[19] Randle, V., and Engler, O., 2000, Introduction to Texture Analysis, Macrotexture, Microtexture \& Orientation Mapping, Gordon and Breach Science, Amsteldijk, The Netherlands, pp. 61-70.

[20] http:/www.panalytical.com/index.cfm?pid-882.

[21] Hurley, P. J., and Humphreys, F. J., 2003, "The Application of EBSD to the Study of Substructural Development in a Cold Rolled Single-Phase Aluminium Alloy," Acta Mater., 51, pp. 1087-1102.

[22] Korbel, A., Embury, J. D., Hatherly, M., Martin, P. L., and Erbsloh, H. W., 1986, "Microstructural Aspects of Strain Localization in Al-Mg Alloys," Acta Metall., 34(10), pp. 1999-2009.

[23] Aernoudt, E., and Stüwe, H. P., 1970, "Die Endlagen der Verformungstextur, insbesondere bei kubisch-flächenzentrierten Metallen," Z. Metallkd., 61, pp. $128-136$.

[24] Becker, R., Butler, J. F., Hu, H., and Lalli, L. A., 1991, "Analysis of an Aluminum Single Crystal With Unstable Initial Orientation (001)[110] in Channel-Die Compression," Metall. Trans. A, 22A, pp. 45-58.

[25] Havner, K. S., and Chidambarrao, D., 1987, "Analysis of a Family of Unstable Lattice Orientations in (110) Channel Die Compression," Acta Mech., 69, pp. 243-269.

[26] Havner, K. S., 1992, "Finite Plastic Deformation of Crystalline Solids," paperback reprint, Cambridge University Press, North American Branch, New York, p. 112.

[27] Surrel, Y., 1996, "Design of Algorithms for Phase Measurements by the Use of Phase Stepping," Appl. Opt., 35(1), pp. 51-60.

[28] Kamijo, T., and Fujiwara, A., 1991, "Shear Bands in High Purity Al," Scr. Metall. Mater., 25, pp. 949-954.

[29] Wróbel, M., Dymek, S., Blicharski, M., and Gorczyca, S., 1993, "Shear Band
Formation in the Rolled Metals," Arch. Metall., 38(2), pp. 195-203.

[30] Vanderesse, N., Desrayaud, Ch., Girard-Insardi, S., and Darrieulat, M., 2008, "Channel-Die Compression at High Temperature," Mater. Sci. Eng., A, 476, pp. 322-332.

[31] Butler, J. F., Jr., and Hu, H., 1989, "Channel Die Compression of Aluminum Single Crystals," Mater. Sci. Eng., A, 114, pp. L29-L33.

[32] Nakayama, Y., and Morii, K., 1986, "Microstructure and Shear Band Formation in Rolled Single Crystals of Al-Mg Alloy," Acta Metall., 34(7), pp. 17471755 .

[33] Wagner, P., Engler, O., and Lücke, K., 1995, "Formation of Cu-Type Shear Bands and Their Influence on Deformation and Texture of Rolled (112)[11 $\overline{1}]$ Single Crystals," Acta Metall. Mater., 43(10), pp. 3799-3812.

[34] Jasieński, Z., Baudin, T., Piątkowski, A., and Penelle, R., 1996, "Orientation Changes Inside Shear Bands Occurring in Channel-Die Compressed (112) $\times[\overline{1} 11]$ Copper Single Crystals," Scr. Mater., 35, pp. 397-403.

[35] Hatherly, M., and Malin, A. S., 1984, "Shear Bands in Deformed Metals," Scr Metall., 18(5), pp. 449-454.

[36] Basson, F., and Driver, J. H., 2000, "Deformation Banding Mechanisms During Plane Strain Compression of Cube-Oriented f.c.c. Crystals," Acta Mater., 48, pp. 2101-2115

[37] Wert, J. M., Liu, Q., and Hansen, N., 1997, "Dislocation Boundary Formation in a Cold Rolled Cube-Oriented Al Single Crystal," Acta Mater., 45, pp. 2565-2576.

[38] Gourdet, S., Jonas, J. J., and Montheillet, F., 1998, “Minimum-Angle Versus Low-Index Axis Rotations for Representing Small- and Large-Angle Grain Boundary Misorientations in Cubic Lattices," J. Appl. Crystallogr., 31, pp. 204-211. 